\title{
A geometrically exact shell theory with initial curvatures and 3D constitutive models
}

\author{
Bruno Aguirre*†£, Carlos Tiago ${ }^{\dagger}$, João R. Correia ${ }^{\dagger}$ and Laurent Stainier $^{\S}$ \\ $\dagger$ Instituto Superior Técnico \\ Av. Rovisco Pais, Lisboa, 1049-001, Portugal \\ $\S$ Institut de Recherche en Génie Civil et Mécanique (GeM) \\ École Centrale de Nantes \\ 1 Rue de la Noe, 44300 Nantes, France \\ *e-mail: bruno.tessaro@tecnico.ulisboa.edu
}

\begin{abstract}
Thin walled structures can be found in most of the major engineering fields, having numerous applications. Describing such strcutures with models based on the full three-dimensional nonlinear theory is inefficient and may lead to locking problems. For the past two centuries, the formulation of shell and plate theories has been a major challenge in the field of structural analysis [1].

Here, we aim to present an initially curved geometrically exact shell model that includes thickness variation and is able to work with large strain problems [2]. In this model, the rotations are analytically parametrized by the 3 parameter Euler-Rodrigues formula. The material points along the thickness are described through a quadratic equation using two transversal degrees of freedom, allowing its deformation. The introduction of thickness variations allows the use of general 3D constitutive models, where no plane stress assumptions are imposed. The model is implemented in a FEM context in conjunction with the Matlib library [3]. This library is composed by numerous constitutive models based on variational incremental updates [4]. Applications of the model containing creep and transverse isotropy are presented.
\end{abstract}

\section{REFERENCES}

[1] C. Tiago, Meshless methods: Extending the linear formulation and its generalization to geometrically exact structural analysis. PhD thesis, Instituto Superior Técnico, Technical University of Lisbon, 2007.

[2] P. Pimenta, E. Campello, and P. Wriggers, "A fully nonlinear multi-parameter shell model with thickness variation and a triangular shell finite element," Computational Mechanics, vol. 34, no. 3, pp. 181-193, 2004.

[3] L. Stainier, F. Dubois, and R. Peyroux, "Matlib, une bibliothèque portable de modèles constitutifs pour la mécanique non-linéaire des solides: concepts et implémentation," in 6ème Colloque National en Calcul des Structures, vol. 3, pp. 25-32, 2003.

[4] L. Stainier, "A variational approach to modeling coupled thermo-mechanical nonlinear dissipative behaviors," Advances in Applied Mechanics, vol. 46, pp. 69-126, 2013. 